\title{
Number of autonomous agents in a neural network
}

This paper was downloaded from TechRxiv (https://www.techrxiv.org).

\section{LICENSE}

CC BY 4.0

SUBMISSION DATE / POSTED DATE

05-11-2021 / 09-11-2021

CITATION

Kak, Subhash (2021): Number of autonomous agents in a neural network. TechRxiv. Preprint. https://doi.org/10.36227/techrxiv.16943194.v1

$\mathrm{DOI}$

10.36227/techrxiv.16943194.v1 


\title{
Number of autonomous agents in a neural network
}

\author{
Subhash Kak \\ Oklahoma State University, Stillwater, OK 74078, USA \\ Email: subhash.kak@okstate.edu
}

November 5, 2021

\begin{abstract}
This paper considers several aspects of the relationship between size, structure, speed of propagation and the number of autonomous cognitive agents in a neural network. Whereas, memory and function generation capacities of neural networks with scale invariant structure have been investigated extensively, the number of autonomous agents has not received prior attention. We propose the emergence of the dichotomy of causal and noncausal regions that is related to speed of propagation, in which the autonomous cognitive agents are not bound in a causal relationship with other agents. Arguments are presented for why the count of autonomous agents is best estimated with respect to the dimensionality of the underlying space. The number of autonomous agents obtained for the human brain equals twenty-five, and it is significant that the number in the sub-system modules also turns out to be close to the same value. It is possible that this near equality across layers provides a special uniqueness to the human brain. We argue that the findings of this study will be useful in the design of neural-network based AI systems that are designed to emulate human cognitive capacity.
\end{abstract}

Keywords: autonomous agents, propagation speed, noninteger dimensional spaces, information theory

\section{Introduction}

The optimal capacity of a neural network is estimated in different ways for engineered systems. For example, the capacity of a feedforward neural network has been defined as the number of functions it can compute [1][2]. On the other hand, the memory capacity of a feedback neural network consisting of $n$ neurons with full connectivity of $n^{2}$ weights is approximately equal to $n$ if delta learning is used and considerably more in networks with non-binary neurons [3][4]. In such studies, it is assumed that the control of the network is done by an agent who inputs and takes out the data after the computation is complete, and so this is a model with a single autonomous agent who normally resides outside the network. 
In the "global neuronal workspace" hypothesis, associative perceptual, motor, attention, memory, and other areas interconnect to form a higher-level unified space where information is broadly shared and broadcast back to lower-level processors [5], and there is much experimental evidence in support of this view [6]. It is plausible that the reason behind the limitations of machines in emulating many aspects of biological information processing (e.g. [7][8]) lies in the relatively smaller number of autonomous agents associated with AI systems.

In the hierarchical models of cognition, consciousness has not been located in any specific center of the brain, therefore it must be seen to belong to an abstract space beyond the physical neural structures [9][10], with the autonomous agents mapped to different parts of the architecture [11][12]. This may be done either by considering a brain-inspired architecture with different modules for various agents and sub-agents [13], or a more abstract architecture [14][15]. In the latter case, one must estimate the capacity of the network to support autonomous agents that are able to perform different cognitive tasks.

The number of agents in a complex cognitive network and optimal performance has two contradictory aspects: (i) process information efficiently and as rapidly as possible by increased propagation speeds, and (ii) have as many cognitive agents as possible to be able to operate with agents and sub-agents as required in the execution of complex behavior. They are contradictory because high speeds imply that the influences will spill beyond local regions and interfere with information associated with other agents. Therefore, events in a decentralized cognitive network may be put in causal (with clear past and future distinction) and noncausal (which corresponds to an ambiguous observer-dependent "present" that guarantees autonomy) domains that are generated by the propagation rate of information. Optimality will depend on the appropriateness of the given propagation velocity for the architecture of the network.

The performance of a cognitive network is also of relevance in evolutionary neuroscience for the study of structures of nervous systems [16][17][18]. Neural structures that are able to perform complex behavior must be recursive [19][20] and therefore evolve beyond two dimensions for two-dimensional structures cannot support distant signaling [21].

It has recently been shown that representation of information requires noninteger dimensional structure with an optimal dimensionality of $e(2.71828 \ldots)$ [22][23][24]. Indeed, this is quite close to the observed value for brain structures with estimated best fit that ranges from 2.6 to 2.8 (see [25][26]). Fractal structures are normally seen through the lens of symmetric transformations in physical systems [27], but in view of the fundamental asymmetry associated with form and function in biological 
systems, we must consider asymmetric generators and it is interesting that such structures in a three-dimensional system can have a columnar form.

Viewed from the perspective of brain size, the connections increase in proportion to the square of the number of neurons [28][29]. This is addressed by making brains more modular which reduces the lengths of many connections and helps isolate agents and sub-agents.

This paper presents broad information-theoretic analysis to find the optimal number of cognitive agents that a neural network can support. This analysis is based on recent new results related to optimal representation of data. We show that columnar fractal structures are an efficient way to represent asymmetric development and this is consistent with the structures found in cerebral neocortex.

\section{Propagation velocity and causal and non-causal subparts}

Nerve conduction velocity $(\mathrm{CV})$ is the speed at which electrochemical impulses propagate down a neural pathway and it is central to the ability of individual agents to form largely segregated subparts within the neural network. Conduction velocities are affected by a wide array of factors and demyelinating disease conditions may result in reduced or non-existent conduction velocities. Myelinated neurons conduct at speeds up to $120 \mathrm{~m} / \mathrm{s}$, and the conduction velocity varies from $3-30 \mathrm{~m} / \mathrm{s}$ in parts of the brain, and in brain-wide data, a propagation velocity of $1.0-1.5 \mathrm{~m} / \mathrm{s}$ has been estimated [30].

In cognitive architectures, the repeated cycle of perception, understanding and action selection, which constitutes the cognitive cycle, is basic to discrete cognitions. Empirical and simulation data [31] indicates that an initial phase of perception (stimulus recognition) occurs $80-100 \mathrm{~ms}$ from stimulus onset under optimal conditions. It is followed by a conscious episode (broadcast) 200-280 ms after stimulus onset, and an action selection phase $60-110 \mathrm{~ms}$ from the start of the conscious phase. One cognitive cycle would therefore take 260-390 ms. At conduction velocity of about $1 \mathrm{~m} / \mathrm{s}$ in the brain, and roughly $1 / 3$ of a second for the cognitive cycle, this means that the influence will have time to spread to $1 / 3$ of a meter.

Considering the activity in the neuron with respect to time, the agents are located physically at some separation from each other to make independent processing possible. Although e-dimensionality is optimal, and the processing does not occur at physical locations, one may view the agents to be located in the overarching 3dimensional space together with the time dimension. 
Noninteger dimensionality may be seen from the perspective of a power law related to connectivity [32]. One may take this connectivity to separation $x$ as being given by

$$
p(x) \sim x^{-\gamma}
$$

where $\gamma$ is a parameter whose value is greater than 1 . The main characteristic of this distribution is its heavy-tailed nature and there is a variety of examples of this in Nature [33].

The activity lines in the e-dimensional space may be seen as in the simplified diagram of Figure 1 which shows the variation of activity for a single "distance" coordinate axis $\mathrm{x}$ with respect to time.

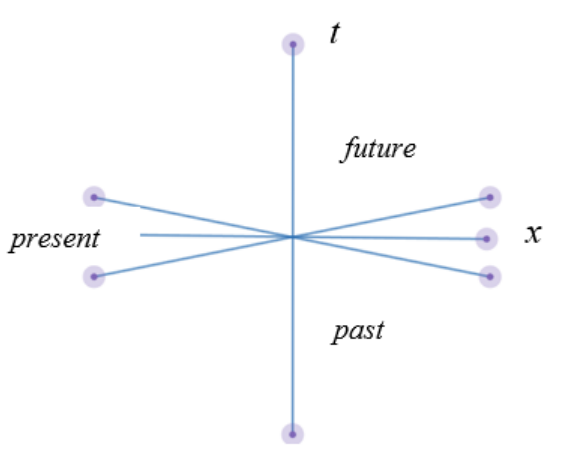

a) Conduction velocity high

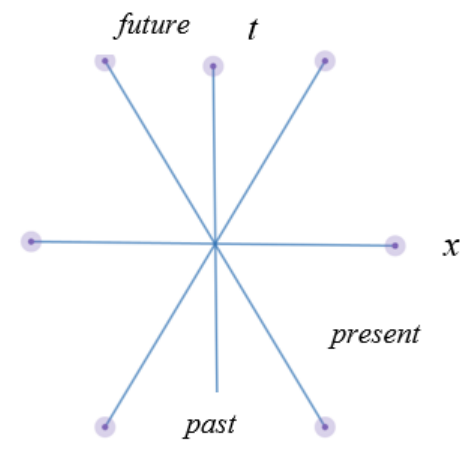

b) Conduction velocity low

Figure 1. Activity lines in the neurons create a sectoral past-future divide leaving other activities to belong to the isolated present where other agents can be located

This activity flow divides up the activity lines for all neurons. If the speed was too high the excitation would resonate in the entire brain quickly and swamp independent processes, and if it were too low, it will not be able to reach across the brain and there is no divide of past and present and the system is not integrated into one overarching unity. Therefore, an intermediate value of conduction velocity is needed.

For the subnetworks associated with sub-agents to remain largely separate, a reduction in the connectivity between them will be helpful. From an evolutionary perspective, an enlargement of a two-dimensional network into the third dimension, as in the columnar architecture of the neocortex [18], seems to be particularly suited to this purpose.. 


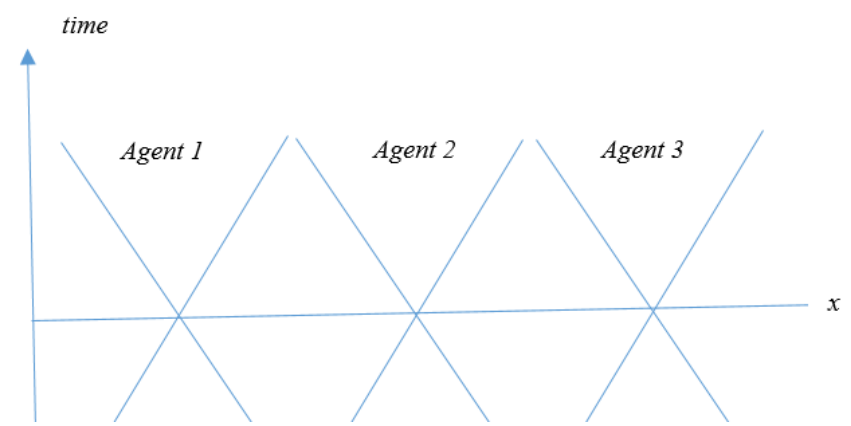

Figure 2. Three different agents within the physical network

For other issues related to optimal connectivity, consider length L connecting $n$ units distributed over volume $\mathrm{V}$, with each unit occupying average volume $V / n$. A tree that optimizes wiring will tend to connect points to their nearest neighbors, which are on average located at distances proportional to $\left(\frac{V}{n}\right)^{d / 3}$. We need at least $n$ such dendritic sections to make up the tree. The total length $\mathrm{L}$ of these sections sums up to

$$
L=\operatorname{cn}\left(\frac{V}{n}\right)^{d / 3}=c V^{d / 3} n^{2 / 3}
$$

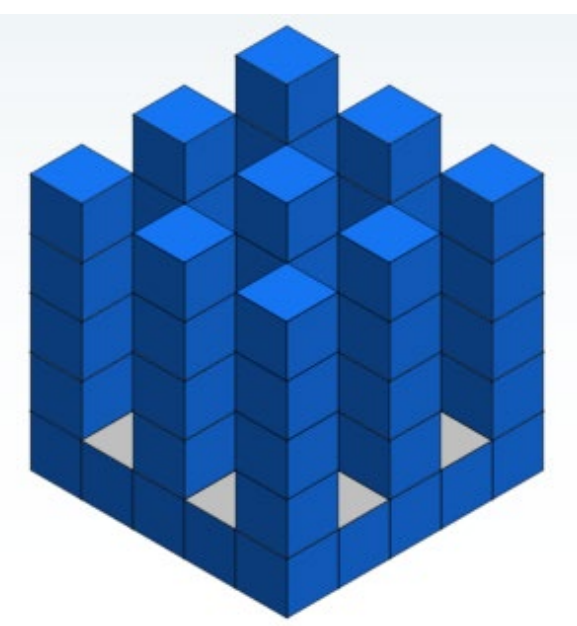

Figure 3. A $5 \times 5 \times 5$ fractal with columnar structure (one iteration shown). The next iteration will have the same structure telescoped into each cube, and the appearance of minicolumns

Knowing that the brain structures are fractal, the search for optimal generators that map the organization of the brain in some approximate sense remains a significant research problem. Here we describe some ad hoc generators that provide a dimensionality between 2 and 3 , as is true for the brain \{25][26].

Figure 3 presents a fractal in which a cube gets transformed into a $5 \times 5$ square surface together with columns. In the next iteration each cube of the diagram will in turn be transformed at a scale that is $1 / 25^{\text {th }}$ of what is shown above. Since the $5 \times 5 \times 5$ 
transformed solid has only 61 sub-cubes $(25+3 \times 12)$ the dimensionality associated with such a recursive function will be obtained easily (as in [33] where a similar computation is done for many examples):

$$
d=\frac{\ln 61}{\ln 5} \cong 2.554
$$

For a different example in the same style as above, see Figure 4.
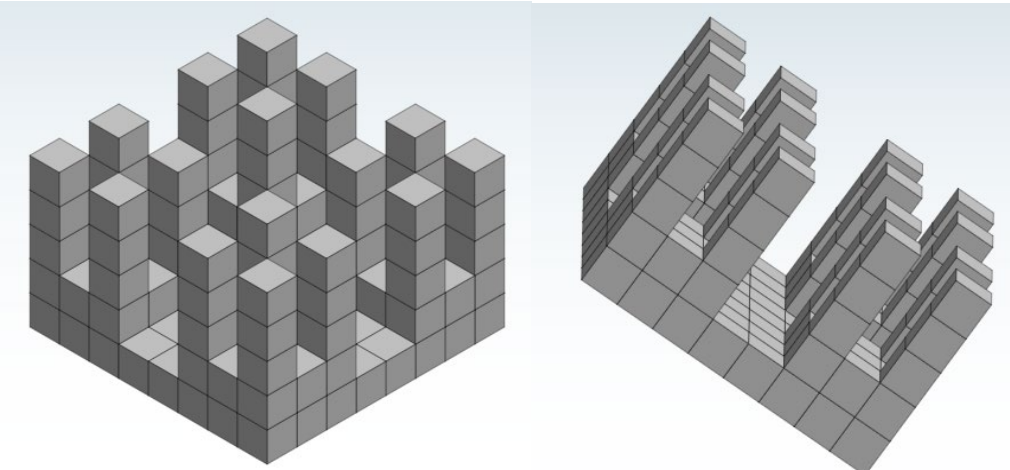

Figure 4. A $8 \times 8 \times 5$ fractal with columnar structure (one iteration shown) (left), and the columns shown clearly (right); further iterations lead to mini- and micro-columns

The number of sub-cubes on the $8 \times 8$ base is $64+4 \times 9+16 \times 3=148$. Hence, the dimensionality of the mapping of Figure 4 will be:

$$
d=\frac{\ln (148)}{\ln (8)} \cong 2.403
$$

Another example of this is the recursive structure of Figure 5 below:

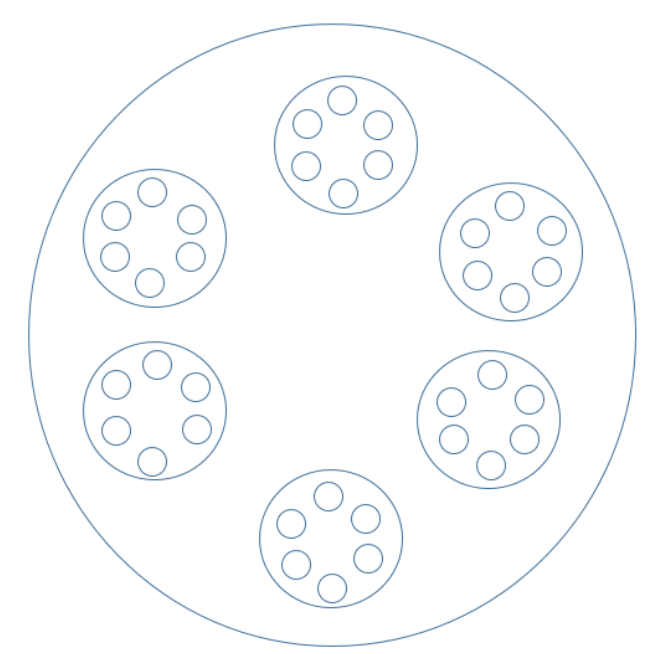

Figure 5. Columns and minicolumns 
Its dimensionality will depend on the relative diameters of the various columns.

\section{Columnar structure in the neocortex}

Optimal fractal mappings for the brain depend on many local constraints defined within the framework of the overall architecture. Thus the mammalian cerebral cortex is a cellular sheet composed of pyramidal and local circuit neurons (or interneurons) deployed in horizontal layers, intersected by vertical (or radial) columns that are stereotypically interconnected in the vertical dimension and share extrinsic connectivity [18]. It is remarkable that the neurons migrate to the cortex after they have been generated. They emerge in ventricular (VZ) and subventricular zones (SVZ)), acquiring positions through long-distance radial and tangential migration across the intermediate zone [34].

The broad layered structure of the neocortex (layers I through VI) is segregated principally by cell type and neuronal connections [34][35]. Layer IV is the main recipient of incoming sensory information and the information is distributed from here to the other layers that indicates both the local and global elements of the connections. The neurons in the upper layers II and III project their axons to other areas of neocortex, whereas those in the deeper layers V and VI often project out of the cortex. Neurons in layer IV receive the majority of the connections from outside the cortex, and themselves make short-range, local connections to other cortical layers. Layer IV is small or missing in the primary motor cortex,

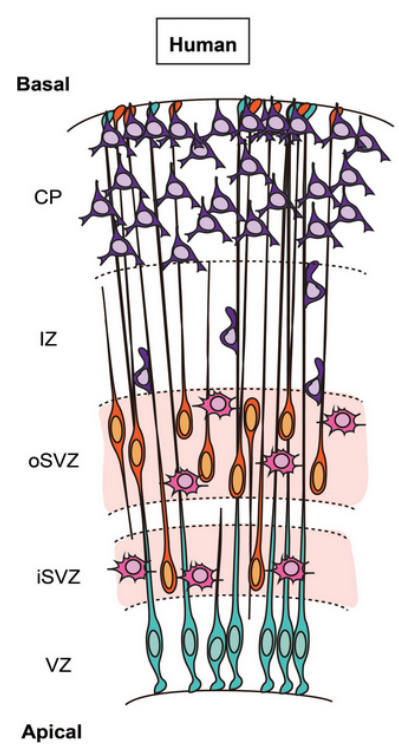

Figure 6. Schematic representation of the columnar cytoarchitecture of the developing neocortex. There are four major zones at mid-neurogenesis, which are the ventricular zone (VZ), the subventricular zone (SVZ), the intermediate zone (IZ), and the cortical plate (CP). (from Pinson et al (2019) [37] 
Because of recursive structure, the architecture is associated with mini- and microcolumns at deeper level. Neurons within the microcolumn receive common inputs, have common outputs, are interconnected, and may "well constitute a fundamental computational unit of the cerebral cortex" [37]. Minicolumns typically have 80-120 neurons, and there are about $2 \times 10^{8}$ minicolumns in the human brain. The diameter of a minicolumn is about $28-40 \mu \mathrm{m}$, with about 100 neurons per minicolumn, yielding an estimate of $2 \times 10^{8}$ minicolumns. From a mathematical point of view, these many minicolumns will be generated by a function that goes through about six or seven recursive steps.

To put these numbers in perspective, it has been estimated that the total length of the average neuron's axonal tree is between 10 and $40 \mathrm{~mm}$, and that the average dendritic tree is $4 \mathrm{~mm}$. As for connectivity, the brain of a three-year-old child has about $10^{15}$ synapses, and this number declines with age, stabilizing by adulthood to number varying from from $10^{14}$ to $5 \times 10^{14}$ synapses.

A biologically plausible scale-invariant neural network is associated with the power law. If $f(x)$ represents the structure or dynamics of a scale-invariant system as a function of the (spatial or temporal) variable $x$, then

$$
f(\lambda x)=C(\lambda) f(x)
$$

Thus if a scale differs from the original one by a factor $\lambda$, replacing $f(x)$ for $f(\lambda x)$, the property described by $f$ remains essentially unchanged; it is only multiplied by a scaling factor $C(\lambda)$ that does not depend on $x$. Although, in principle, $C(\lambda)$ can be any function, in practice, it is a power law for most natural systems. Therefore, we can write

$$
f(\lambda x)=\lambda^{\delta} f(x)
$$

Scale-invariant structures can characterize systems in which physical space does not play an important role. Thus, scale-invariance can be given by topological distance rather than physical distance, and it may then be related to the number of steps required to connect different parts of the system via interaction links [36].

Let the cost of connectivity of a neuron to another in layer $k$ be $C(k)$. Then

$$
C(k+1)=C(k) \frac{(k+1)}{k}+\frac{r(k+1)}{r(k)}
$$


In other words, the cost of layer $(k+1)$ must be at least $\frac{(k+1)}{k}$ times the cost of connectivity to layer $k$, and $\frac{r(k+1)}{r(k)}$ is the additional cost ratio in the next layer related to additional processing associated with signal delays. Both these taken together may be taken to be the origin of the power law expression of equation (1).

The neocortex is divided, into regions demarcated by the cranial sutures into frontal, parietal, occipital, and temporal lobes that perform different cognitive functions with further subdivisions for more specific cognitive processes.

\section{Number of independent centers}

Although it is conventional to represent computer information in terms of bits, this is strictly correct only for two-dimensional surfaces. The number of independent centers for a two dimensional surface with $\mathrm{N}$ points is $\log _{2} N$. This is clear from the example that 16 points on a two-dimensional surface can be labeled $0000,0001, \ldots .1111$. So whereas for memory this offers 16 possibilities, as far as agency within the network is concerned, each of the four places can autonomously fill in the binary data at that point. The logarithm is to base 2 because we have implicitly chosen a two-dimensional surface.

In general for a $d$-dimensional space, the number of independent centers with $\mathrm{N}$ points is

$$
\mathrm{r}=\log _{d} N
$$

We have already mentioned that the optimal representation of data is in an $e$ dimensional space [23], with some recently published applications [38][39]. But we cannot be sure that the brain neural structures correspond to this dimensionality because there are additional factors such as the underlying quantum processes that are ignored [40]. Nevertheless, the abstract space in which genetic information is expressed also appears to satisfy $e$-dimensionality [41][42].

The human brain consists of about 86 billion neurons [43] that are estimated to be connected by $850,000 \mathrm{~km}$ of axons and dendrites. Of this total, roughly $80 \%$ is shortrange, local connections (averaging 680 microns in length), and approximately $20 \%$ is long-range, global connections in the form of myelinated fibers several $\mathrm{cms}$ in length. If we were to consider the fractal dimensionality of the brain to be approximated by the optimal value of $e$, which is not very different from the estimated best fit of 2.811 [44][45], the number of independent cognitive agents in the brain will be approximately 


$$
\log _{e} 86 \times 10^{9} \approx 25
$$

One can assume that each agent is associated with a separate cognitive category associated with a unique abstraction. Intuitive sense may have led ancient philosophers to pick this as the number of categories associated with the mind, as in Sānkhya [46]. One could argue that this number was arrived at introspectively by means of consciousness enumerating its several projections.

Since the neocortex has about 19 percent of the total number of neurons, number of cognitive agents associated with the neocortex will be:

$$
\log _{e} 0.19 \times 86 \times 10^{9} \approx 23.6
$$

A similar count will be associated with number of agents in other modules with billion neuron structures.

That the number of agents saturates very rapidly as the billion neuron range is reached may very well be an explanation for the size of the human brain.

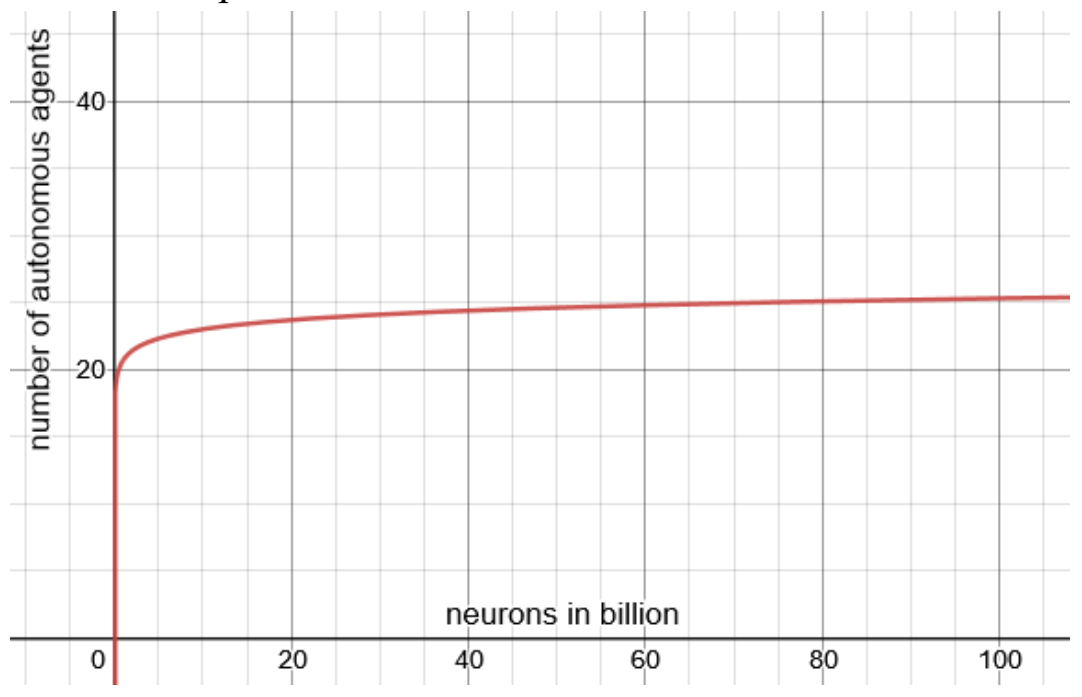

One may speculate that this characteristic of comparable number of autonomous agents at different levels is of particular significance in information processing and cognition made by the brain.

\section{Conclusions}

This paper analyzed the relationship between size, structure, speed of propagation and the number of cognitive agents sharing the resources in brains. The idea of timerelated and space-related events was defined and related to the speed of propagation, 
in which the autonomous cognitive agents are space-related. We considered the number of autonomous agents a neural network with scale invariant structure can support. It was shown that the number of agents and sub-agents is about 25 , which is quite consistent with analysis using linguistic and philosophical categories. Ad hoc fractal generators that lead to columns and minicolumns were proposed and these and other generators could be further investigated for their mathematical properties.

It was shown that the number of autonomous agents flattens out in the billion neuron range which creates the possibility that the number of autonomous agents is more or less the same across different modules. This fact could very well be a factor in the evolution of the brain.

\section{REFERENCES}

1. Baldi, P. and Vershynin, R. The capacity of feedforward neural networks. arXiv:1901.00434 (2019)

2. Guliyev, N. Ismailov, V. Approximation capability of two hidden layer feedforward neural networks with fixed weights. Neurocomputing 316, 262-269 (2018)

3. Prados, D. and Kak, S. Neural network capacity using the delta rule. Electronics Letters 25, 197-199 (1989)

4. Prados, D. and Kak, S. Non-binary neural networks. Lecture Notes in Computing and Control 130: 97-104, 1989.

5. Dehaene, S., Kerszberg, M. and Changeux, J.-P. A neuronal model of a global workspace in effortful cognitive tasks. PNAS 95 (24), 14529-14534 (1998); https://doi.org/10.1073/pnas.95.24.14529

6. Deco, G., Vidaurre, D, and Kringelbach, M.L. Revisiting the global workspace orchestrating the hierarchical organization of the human brain. Nature Human Behaviour (2021). DOI: 10.1038/s41562-020-01003-6

7. Reason, C.M. Consciousness is not a physically provable property, Journal of Mind and Behavior 37 (1),31-46 (2016)

8. Kak, S. The limits to machine consciousness. Journal of Artificial Intelligence and Consciousness 8, (2021)

9. Kak, A. et al. A three-layered model for consciousness states. NeuroQuantology 14, 166-174 (2016); doi: 10.14704/nq.2016.14.2.935

10. Felleman, D. J. \& Van Essen, D. C. Distributed hierarchical processing in the primate cerebral cortex. Cereb. Cortex 1, 1-47 (1991)

11. Markov, N. T. et al. Anatomy of hierarchy: feedforward and feedback pathways in macaque visual cortex. J. Comp. Neurol. 522, 225-259 (2014) 
12. Bullmore, E. \& Sporns, O. The economy of brain network organization. Nat. Rev. Neurosci. 13, 336-349 (2012)

13. van den Heuvel, M. P. \& Sporns, O. Rich-club organization of the human connectome. J. Neurosci. 31, 15775-15786 (2011)

14. Chella, A., Frixione, M., Gaglio, S. A cognitive architecture for robot selfconsciousness. Artificial Intelligence in Medicine 44, 147-154 (2008)

15. Gamez, D. Human and machine consciousness. Open Book Publishers (20188)

16. Kaas, J.H. Why is brain size so important. Brain and Mind 1, 7-23 (2000). https://doi.org/10.1023/A:1010028405318

17. Platek, S.M., Shackelford, T.K. Foundations in Evolutionary Cognitive Neuroscience. Cambridge University Press (2009)

18. Mountcastle, V.B. The columnar organization of the neocortex. Brain 120, 701722 (1997)

19. Martins M. D. Distinctive signatures of recursion. Philosophical transactions of the Royal Society of London. Series B, Biological sciences 367(1598), 20552064 (2012); https://doi.org/10.1098/rstb.2012.0097

20. Kak, S. The Loom of Time. DK Printworld, New Delhi (2016)

21. Kak, S. Asymptotic freedom and noninteger dimensionality. Scientific Reports 11, 1-5 (2021); https://www.nature.com/articles/s41598-021-83002-9

22. Kak, S. Information theory and dimensionality of space. Scientific Reports 10 , 20733, (2020)

23. Kak, S. The intrinsic dimensionality of data. Circuits Syst. Signal Process. 40,2599-2607 (2021); https://doi.org/10.1007/s00034-020-01583-8

24. Kak, S. Fractals with optimal information dimension. Circuits Syst. Signal Process. 40, 5733-5743 (2021); https://link.springer.com/article/10.1007/s00034-021-01726-5

25. Krohn, S. et al. Evaluation of the 3D fractal dimension as a marker of structural brain complexity in multiple-acquisition MRI. Human Brain Mapping 40, 32993320 (2019)

26. Reishofer, G., Studencnik, F., Koschutnig, K. et al. Age is reflected in the fractal dimensionality of MRI diffusion based tractography. Sci Rep 8, 5431 (2018). https://doi.org/10.1038/s41598-018-23769-6

27. Kak, S. Two classes of regular symmetric fractals. TechRxiv (2021); https://doi.org/10.36227/techrxiv.14714094.v2

28. Herculano-Houzel, S. The human brain in numbers: a linearly scaled-up primate brain. Frontiers in human neuroscience, 3, 31 (2009); https://doi.org/10.3389/neuro.09.031.2009

29. Siegel, A. and Sapru, H.N. Essential Neuroscience. Lippincott Williams \& Wilkins (2006).

30. Shimono, M., Hatano, N. Efficient communication dynamics on macroconnectome, and the propagation speed. Sci Rep 8, 2510 (2018) 
31. Madl, T., Baars, B. J., \& Franklin, S. The timing of the cognitive cycle. PloS one, 6(4), e14803. (2011); https://doi.org/10.1371/journal.pone.0014803

32. Tomasi, D.G., Shokri-Kojori, E., Volkow, N.D. Brain network dynamics adhere to a power law. Front. Neurosci. (2017); https://doi.org/10.3389/fnins.2017.00072

33. Falconer, K. J. Fractal Geometry: Mathematical Foundations and Applications. Wiley (2003)

34. Cuntz, H., Mathy, A., Häusser, M. A scaling law derived from optimal dendritic wiring. PNAS 109, 11014-11018 https://doi.org/10.1073/pnas.1200430109

35. Rakic, P. Evolution of the neocortex: a perspective from developmental biology. Nature Reviews Neuroscience 10, 724-735 (2009)

36. Yara, $K$. et al. Scale invariance in natural and artificial collective systems: a review. J. R. Soc. Interface 14, $20170662-20170662$ (2017)

37. Buxhoeveden, D.P., Casanova, M.F. The minicolumn hypothesis in neuroscience. Brain. 125, 935-951 (2002)

38. Kak, S. Information theory and dimensionality of space. Scientific Reports 10, 20733 (2020). https://doi.org/10.1038/s41598-020-77855-9

39. Kak, S. The base-e representation of numbers and the power law. Circuits Syst. Signal Process. 40, 490-500 (2021); https://doi.org/10.1007/s00034-020-014800

40. Kak, S. Active agents, intelligence and quantum computing. Information Sciences 128, 1-17 (2000)

41. Kak, S. The e-Dimensionality of genetic information. TechRxiv (2021); https://doi.org/10.36227/techrxiv.14977479.v1

42. Kak, S. Data amplification using the iterated Newcomb-Benford distribution. TechRxiv. (2021); https://doi.org/10.36227/techrxiv.15832083.v1

43. Pinson, A., Namba, T., Huttner, W.B. Malformations of Human Neocortex in Development - Their Progenitor Cell Basis and Experimental Model Systems. Front. Cell. Neurosci., (2019) | https://doi.org/10.3389/fncel.2019.00305

44. Azevedo, F.A., Carvalho, L.R., Grinberg, L.T., et al. Equal numbers of neuronal and nonneuronal cells make the human brain an isometrically scaled-up primate brain. J Comp Neurol. 2009 Apr 10;513(5):532-41. doi: 10.1002/cne.21974. PMID: 19226510.

45. Kriegeskorte, N., Douglas, P.K., Cognitive computational neuroscience. Nature Neuroscience 21, 1148-1160 (2018)

46. Larson, G.J., Bhattacharya, R.S., Potter, K. The Encyclopedia of Indian Philosophies, Volume 4. Princeton University Press (2014) 